\title{
F-actin distribution pattern in the nuclei of early mouse embryos
}

\section{Irina Bogolyubova}

\author{
Institute of Cytology, Russian Academy of Sciences, 194064 St. Petersburg, Russia
}

\begin{abstract}
Nuclear actin is the essential component of gene expression. Here we show that the pattern of F- actin distribution in the nuclei of early mouse embryos depends on the experimental conditions and does not represent nonspecific cell reaction for the experimental influence.
\end{abstract}

Key words: Early mouse embryo -nuclear domains - transcription - nuclear actin

\section{Introduction}

It is generally assumed now that nuclear actin is the essential component of gene expression (Pederson, Aebi, 2005; Pederson, 2008). Modern studies have demonstrated nuclear actin to take part in many basic nuclear processes including transcription (Miralles, Visa, 2006). The presence of classical fibrillar (F-) actin in the nucleus, however, is still far from convincing (Pederson, 2008). When transcription is suppressed, F-actin forms a network around chromosomes (Scheer et al., 1984). Similar effect is provoked by dimethyl sulphoxide treatment (Wehland et al., 1980) or by heat-shock (Welch, Suhan, 1985). It is unclear whether the presence of Factin in the nucleus is the specific response to changes of the transcriptional activity or it appears nonspecifically under suboptimal condition of cell culturing. Early mammalian embryos are very sensitive to the environment and, thus, may serve as an appropriate model to study Factin distribution in the nuclei under different culture conditions. In this work we used the following groups of embryos: (i) control ones immediately fixed after explantation, (ii) cultured in vitro in a standard medium, (iii) treated with an inhibitor of transcription, and (iv) kept under intentionally unfavorable conditions.

\section{Materials and methods}

Inbred BALB/c mice obtained from the animal nursery "Rappolovo" of the Russian Academy of Medical Sciences were used.

Correspondence: Irina O. Bogolyubova, Lab. of Cell Morphology, Institute of Cytology RAS. Tikhoretsky avenue 4, 194064, St. Petersburg, Russia; e-mail: ibogol@mail.ru
Superovulation was induced and the embryos were collected and cultured as described earlier (Bogolyubova et al., 2006). To inhibit transcription, embryos were treated with 5,6-dichloro-1- $\beta$-Dribofuranosylbenzimidazole (DRB; BIOMOL Research Labs). A 5 $\mu \mathrm{g} / \mathrm{ml}$ stock solution of the dry powder was prepared in $96 \%$ ethanol and then diluted with the incubation medium. The embryos were incubated in $\sim 500 \mu \mathrm{M}$ DRB for $3 \mathrm{~h}$ and $8 \mathrm{~h}$. To provoke degeneration of the embryos, they were kept in F10 medium for 3 $\mathrm{h}$ at room temperature without $\mathrm{CO}_{2}$ environment.

Embryos were fixed for $1.0 \mathrm{~h}$ in $4 \%$ paraformaldehyde in PBS, washed in PBS and treated for $1 \mathrm{~h}$ by $10 \mu \mathrm{M} / \mathrm{ml}$ TRITC-phalloidin (Sigma). After rinsing in PBS, the preparations were mounted in Vectashield (Vector Laboratories, USA). The samples were examined with a Leica TSC SL confocal laser scanning microscope (Heidelberg, Germany). Contrast and relative intensities of images were adjusted with Adobe Photoshop.

\section{Results and discussion}

In control 2-cell embryos developing in vivo we revealed no significant amount of F-actin in the nuclei. Noticeable staining was observed only in the peripherical zone of the cytoplasm and in a zone of contact between blastomeres (Fig. 1). Similar pattern of staining characterized the main portion of embryos cultured in vitro for $3 \mathrm{hrs}$ (Fig. 2), but not for $8 \mathrm{hrs}$. In the latter case, clear but not very intensive fluorescence was registered in the nuclei of $\sim 50 \%$ of studied embryos (Fig. $3)$. It should be noted that the nuclei were diffusely stained with the exception of nucleolar precursor bodies (NPBs) (Fig. 3, arrows). More intensive staining was observed in the nuclei of the majority of polar bodies (PBs); at the same time, the intensity of peripherical fluorescence was decreased as compared to the control.

The nuclei of the main part of embryos treated for 3 hrs by DRB, an inhibitor of kinases that phosphory- 

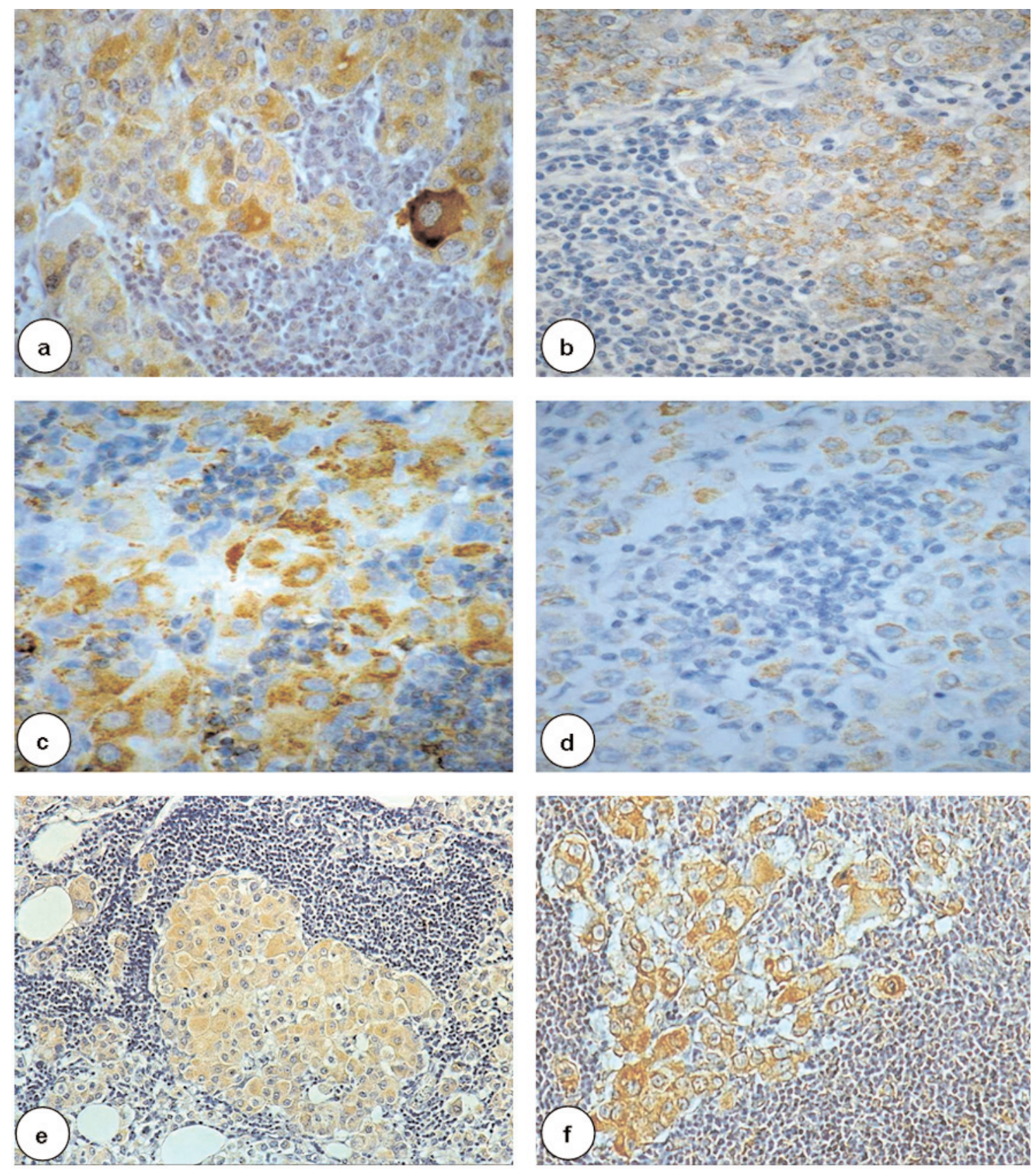

Figs 1-6. Distribution of F-actin in late 2-cell mouse embryos. 1 - a control embryo developing in vivo; 2, 3 - control embryos cultured in vitro for 3 and $8 \mathrm{hrs}$, respectively; 4 - an embryo incubated for $3 \mathrm{hrs}$ under non-physiological condition; 5, 6 - embryos cultured with DRB for 3 and 8 hrs, respectively. Arrows indicate nucleolar precursor bodies. Bar $=20 \mu \mathrm{m}$.

late the C-terminal domain of RNA polymerase II, were not noticeable stained (Fig. 5). In this experimental group, we registered low fluorescence of cytoplasm, especially in the perinucleolar part. After $8 \mathrm{hrs}$ of DRB treatment the pattern of labeling was changed in $\sim 50 \%$ embryos. The fluorescence appeared in the nuclei of blastomeres and PBs, but the intensity of peripherical labeling was decreased (Fig. 6). Under these conditions, the NPBs were noticeably stained in blastomeres, but not in the PBs. In the embryos degraded in unfavorable conditions, neither nucleoplasm nor NPBs were stained (Fig. 4).

Thus, our data suggest that the distribution pattern of F-actin in the nuclei of early mouse embryos depends on the experimental conditions and does not represent nonspecific cell reaction for the experimental influence. The appearance of F-actin in association with chromatin might be caused by the incubation of embryos in vitro under suboptimal condition. In our work we used M3 medium that does not contain 
growth factors and other components essential for normal embryo development. It is known that culture mediums like M3 are not able to support normal preimplantation development of some animal strains (Telford et al., 1990). Some authors suggest that the absence of F-actin in the nuclei might be explained by insufficient concentration of monomeric nuclear actin (Clark, Rosenbaum, 1979). The accumulation of Gactin in the nucleus of physiologically "unhappy" cells permits actin polymerization. This hypothesis explains the appearance of the F-actin in the nuclei of PBs that represent the classical example of degrading cells. However, it should be noted that the beginning of actin polymerization in the nucleus requires the define time. So, we never observed the accumulation of nuclear Factin in cells that are kept under unfavorable conditions for $3 \mathrm{hrs}$ despite of clear evidences of their degradation (Fig. 4).

The appearance the F-actin in NPBs after DRB treatment seems to be a response to transcription inhibition although this supposition needs to be verified. In the nuclei of transcriptionally arrested cells under physiological or experimental conditions many molecular components of gene expression are known to redistribute to heterogenous extrachromosomal nuclear domains (Biggiogera, Pellicciari, 2000; Bogolyubov, Parfenov, 2008). The accumulation of Factin in NPBs of mouse embryos after DRB treatment resembles this situation.

In the end, the appearance of F-actin in the nuclei is not a quick response to the alterations of environmental conditions that influence its distribution.

Acknowledgements: The author thanks Dr. D.Bogolyubov for critical reading of the manuscript. This work was supported by Russian Foundation for Basic Research (grants 07-04-00685 and 09-04-00723) and by the grant program of RAS "Molecular and cell biology".

\section{References}

[1] Biggiogera M, Pelliciari C. Heterogeneous ectopic RNPderived structures (HERDS) are markers of transcriptional arrest. FASEB J. 2000;14:828-834.

[2] Bogolyubov D, Parfenov V. Structure of the insect oocyte nucleus with special reference to interchromatin granules clusters and Cajal bodies. Int Rev Cell Mol Biol. 2008;263:59110.

[3] Bogolyubova IO, Bogoliubova NA, Bogolyubov DS, Parfenov VN. Nuclear structure in early mouse embryos: a comparative ultrastructural and immunocytochemical study with special emphasis on the "2-cell block in vitro". Tissue Cell. 2006;38:389-398.

[4] Clark TG, Rosenbaum JL. An actin filament matrix in handisolated nuclei of Xenopus laevis oocytes. Cell. 1979;18:1101-1108.

[5] Miralles F, Visa N. Actin in transcription and transcription regulation. Curr Opin Cell Biol. 2006;18:261-266.

[ 6] Pederson T, Aebi U. Nuclear actin extends, with no contraction in sight. Mol Biol Cell. 2005; 16:5055-5060.

[ 7] Pederson T. As functional nuclear actin comes into view, is it globular, filamentous, or both? J Cell Biol. 2008;180:10611064.

[ 8] Scheer U, Hinssen H, Franke WW, Jockusch BM. Microinjection of actin-binding proteins and actin antibodies demonstrates involvement of nuclear actin in transcription of lampbrush chromosomes. Cell. 1984;39:111-122.

[ 9] Telford NA, Watson AJ, Schultz GA. Transition from maternal to embryonic control in early mammalian development: a comparison of several species. Mol Reprod Dev. 1990;26:90100.

[10] Wehland J, Weber K, Osborn M. Translocation of actin from the cytoplasm into the nucleus in mammalian cells expressed to dimethylsulfoxide. Biol Cell. 1980;39:109-111.

[11] Welch WJ, Suhan JP. Morphological study of the mammalian stress response: characterization of changes in cytoplasmic organelles, cytoskeleton, and nucleoli, and appearance of intranuclear actin filaments in rat fibroblasts after heat-shock treatment. J Cell Biol. 1985;101:1198-1211.

Submitted: 7 April, 2009 Accepted after reviews: 17 July, 2009 